\title{
Iatrogenic Dry Eye Disease: Dealing with the Conundrum of Post-Cataract Discomfort. A P.I.C.A.S.S.O. Board Narrative Review
}

\author{
Rita Mencucci · Roberto Vignapiano · Pierangela Rubino • \\ Eleonora Favuzza • Emilia Cantera · Pasquale Aragona • \\ Maurizio Rolando
}

Received: November 25, 2020 / Accepted: January 15, 2021 / Published online: February 8, 2021

(C) The Author(s) 2021

\section{ABSTRACT}

The incidence and prevalence of dry eye disease (DED) after cataract surgery is greatly underestimated. The severity of dry eye symptoms has been reported to peak 7 days after cataract surgery and may persist for months, significantly affecting patients' quality of life (QoL). The importance of considering surgical outcomes not only in terms of visual acuity, but also in terms of the patients' QoL, necessitates the

Pasquale Aragona and Maurizio Rolando contributed equally to this review.

R. Mencucci $(\bowtie) \cdot$ R. Vignapiano · E. Favuzza Eye Clinic, NEUROFARBA Department, University of Florence, Florence, Italy

e-mail: rita.mencucci@unifi.it

P. Rubino

Ophthalmology, University of Parma, Parma, Italy

E. Cantera

Ophthalmology, Israelitic Hospital, Rome, Italy

P. Aragona

Department of Biomedical Sciences,

Ophthalmology Clinic, University of Messina,

Messina, Italy

M. Rolando

Ocular Surface Unit, ISPRE, Genoa, Italy assessment and evaluation of the ocular surface by the cataract surgeon prior to the procedure. This narrative review, drafted by the P.I.C.A.S.S.O. (Italian Partners for the Correction of Ocular Surface Alterations) board, analyses the physiopathology of post-cataract surgery DED and highlights the pre-, intra- and postoperative risk factors that may alter ocular surface homeostasis; it proposes a practical comprehensive algorithm for the prevention, treatment and management of DED associated with cataract surgery. Particular attention needs to be paid to the pre- and intraoperative risk factors to reduce the incidence of postoperative dry eye and to improve cataract surgery outcome.

Keywords: Cataract; Cataract surgery; Dry eye; Dry eye disease; Iatrogenic dry eye; Ocular discomfort; Ocular surface 


\section{Key Summary Points}

The incidence and prevalence of dry eye disease (DED) after cataract surgery is greatly underestimated.

Postoperative DED is a frequent complication of cataract surgery; its prevalence fluctuates from 9.8 and 34\%.

The presence of DED and its risk factors should be investigated before cataract surgery to avoid postoperative complications.

A proactive pathophysiological approach based on $3+2$ method is useful for a global evaluation and treatment of DED in both the pre- and postoperative phase.

\section{DIGITAL FEATURES}

This article is published with digital features, including a summary slide, to facilitate understanding of the article. To view digital features for this article go to https://doi.org/10.6084/ m9.figshare.13573859.

\section{INTRODUCTION}

Cataract surgery is one of the most common and successfully performed ocular procedures worldwide, with an excellent postoperative visual outcome [1]. However, in some cases, patients complain of ocular discomfort and dry eye symptoms, including foreign body sensation, ocular irritation, transient blurred vision, intermittent pain, intolerance to wind and light sensitivity. In addition, studies have shown that patients previously diagnosed with dry eye disease (DED) report a worsening of symptoms after uncomplicated phacoemulsification $[1,2]$.

DED is a common disabling ocular condition with a prevalence ranging between 5 and 50\% in the adult population [3]. However, the exact physiopathology of post-surgical DED has yet to be elucidated. Pre, intra- and postoperative risk factors may alter ocular surface homeostasis resulting in tear film instability, hyperosmolarity and inflammation of the ocular surface [4-7]. Many studies have reported a significant association between meibomian gland dysfunction (MGD) and cataract surgery, with asymptomatic MGD suggested to be twice as prevalent as symptomatic forms $[8,9]$. In addition, corneal sensitivity impairment following cataract surgery has been evidenced [7]. Therefore, lid margin and sensory alterations may play an important role in the inflammatory and immunological tissue response that perpetuates the vicious cycle of postoperative DED [7-9].

In the real-world setting, the prevalence and incidence of DED after cataract surgery are highly underrated. Postoperative DED prevalence fluctuates from 9.8 and 34\% [1]. In 2015, the American Society of Cataract and Refractive Surgery (ASCRS) reported a DED prevalence of around $40 \%$ in patients who underwent cataract surgery. The difficulty in making an accurate diagnosis of DED is often related to the discrepancy between signs and symptoms $[10,11]$.

Moreover, the prevalence of DED in patients submitted to cataract surgery is underestimated as well. Recently, an observational study by Trattler et al. [12] reported that $<25 \%$ of patients undergoing cataract surgery had been previously diagnosed with DED. In the study cohort, $80 \%$ of patients had a tear break-up time (TBUT) $\leq 7$ s while $50 \%$ showed positive corneal central staining. Using point-of-care testing (POCT), Gupta et al. [13] revealed that in a sample of patients undergoing cataract surgery, the preoperative incidence of ocular surface disfunction was $>80 \%$, whilst $>50 \%$ of asymptomatic patients had an abnormal tear osmolarity or matrixmetalloproteinase-9 (MMP9) level.

The severity of dry eye symptoms has been reported to peak 7 days after cataract surgery and may persist for months [10, 14, 15]. This can damage the patients both physically and psychologically and have a negative influence on their quality of life (QoL) [10, 14, 15]. Therefore, although the surgical procedure may have been performed correctly, many patients attribute these symptoms and a subsequent decrease in QoL to complications arising from 
the cataract surgery. An impaired ocular surface might also adversely affect preoperative planning, including intraocular lens (IOL) calculation, and lead to refractive errors and postoperative higher order aberrations [16]. These factors contribute to an unsatisfactory postoperative visual recovery and a perceived negative outcome on the patient QoL [17].

The importance of considering surgical outcomes not only in terms of visual acuity but also in terms of the patients' QoL necessitates the assessment and evaluation of the ocular surface by the cataract surgeon prior to the procedure. This article, based on the existing literature and on a consensus among the members of our study group composed of dry eye experts (the P.I.C.A.S.S.O. board, Italian Partners for the Correction of Ocular Surface Alterations), proposes a practical algorithm to identify and manage patients affected by ocular surface disease who undergo cataract surgery through the evaluation of risk factors, correct diagnosis and an appropriate therapeutic strategy [18].

\section{METHODS}

A literature search using various databases, including PubMed, Scopus and ScienceDirect, was conducted for studies and reviews published in English. No restrictions were set for the date of publications, which ranged from 1985 to 2020. Key search terms included "postoperative dry eye", "iatrogenic dry eye" and a combination of the terms "cataract surgery" and "dry eye". Inclusion criteria for publications included those that discussed the aetiologies, risk factors, pathogenesis, clinical manifestations, prophylaxis and management of post-cataract surgery dry eye disease. References were also acquired from citations in publications found in the original search. Two independent authors (R.M. and R.V.) selected the relevant articles for this review article.

This article is based on previously conducted studies and does not contain any studies with human participants or animals performed by any of the authors.

\section{PATHOPHYSIOLOGY OF IATROGENIC DRY EYE DISEASE}

Cataract surgery is considered an ocular surface damaging procedure, with DED the most common complication [15], potentially distressful for our patients. In DED, tear hyperosmolarity and tear film instability stimulate a cascade of events on the ocular surface with consequent production of inflammatory cytokines, interleukins and proteases [9, 19-21]. The recruitment of inflammatory cells represents an additional source of inflammatory mediators, which leads to apoptotic death of surface epithelial cells, loss of goblet cells and reduced expression of glycocalyx mucins [9, 19-21]. This, together with a physiological decrease in lacrimal production, common in elderly people, initiates or amplifies ocular surface hyperosmolarity, establishing the mechanism that perpetuates DED [9, 19-21].

Many different pathophysiological pathways can induce or aggravate DED pre-, intra- and/or postoperatively (Fig. 1). For example, preoperative topical anaesthetics can cause an impairment of the ocular surface in terms of direct toxicity to the corneal epithelium, stroma and

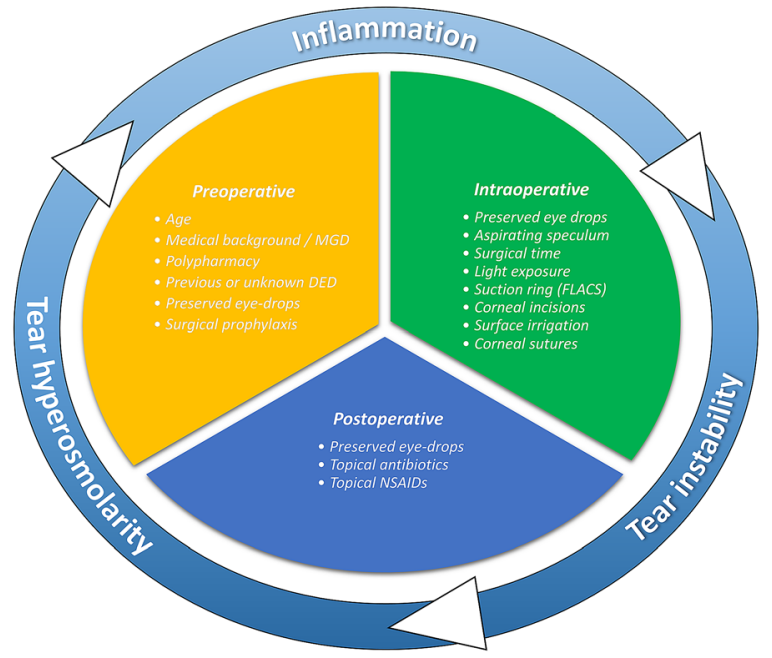

Fig. 1 Vicious cycle of DED related to cataract surgery and its associated pre-, intra- and postoperative risk factors. $N S A I D s$ nonsteroidal anti-inflammatory drugs, DED dry eye disease, $M G D$ meibomian gland dysfunction 
endothelium, whilst topical mydriatics have been reported to cause a higher incidence of blepharitis and drug-induced ocular surface inflammation, which may result in tear film changes leading to a higher risk of DED $[22,23]$. These problems can be further aggravated if the topical anaesthesia or mydriatic eye drops contain preservatives such as benzalkonium chloride (BAK) $[24,25]$. It is well documented that BAK causes dose-dependent conjunctival and corneal epithelial cell toxicity in vivo and in vitro, tear film instability and corneal epithelial barrier dysfunction and may also enhance transcorneal permeability of ophthalmic drugs [24, 25]. Topical application of BAK to the eye has also been reported to trigger corneal neurotoxicity, inflammation and reduced aqueous tear production, contributing to postoperative DED. Corneal epithelial dysfunction occurs even when the concentration of BAK is low $(0.001 \%)$ if it is used frequently or for over a period of $30 \mathrm{~min}$ [24, 25].

Intraoperatively, physiological defence mechanisms such as blinking or miosis cannot perform their normal roles, exposing the ocular structures to photo-thermal, photo-mechanical and photo-chemical alterations. Light exposure from the operating microscope may also be a factor that contributes to postoperative DED $[26,27]$. In vitro studies $[26,27]$ have shown that ocular surface changes after irradiation are consistent with DED, manifesting as tear deficiency, loss of conjunctival goblet cells and decreased mucin production. An increase in IL$1 \beta$, one of the markers of inflammation, suggests that light exposure has phototoxic effects stimulating the production of reactive oxygen species (ROS) in various cell types. Moreover, in conjunctival cells, light exposure might slow down the wound healing and reduce their viability $[26,27]$. Whilst surgical time is typically 10-15 min and light exposure time may be shorter, it is widely recognised that cataract surgery represents an ocular surface trauma inducing inflammation [28]. Therefore, the longer the operative time, the greater the inflammatory response and the more damage that can potentially be caused to the ocular surface and goblet cell density. Consequently, to prevent postoperative DED, it is important to shorten the operative time and exposure to microscope light [6].

Postoperatively, several studies have evidenced a significant impairment in corneal sensitivity after phacoemulsification mainly in the central part of the cornea $[6,29]$. As corneal sensation is partially responsible for tear secretion, an impairment in corneal sensitivity affects tear production through a feedback mechanism between the cornea and the lacrimal gland. After cataract surgery, alteration in corneal sensitivity is thought to be dependent on the extent of the corneal incision $[6,29]$. A greater incision size corresponds to a slower recovery of corneal sensitivity and wound healing (up to 3 months in $4.1 \mathrm{~mm}$ corneal incisions [29]), whilst a smaller incision is related to a focal decrease in corneal sensitivity, quicker recovery (1 month) [6] and minimal effect on tear film stability, probably due to the transection of fewer nerve fibres. The role of incision shape and location on corneal sensitivity and dry eye symptoms has also been evaluated [30]. A significant risk of developing dry eye symptoms was observed in patients with grooved incisions. Concerning the location of the incision, no differences were observed in the postoperative dry eye test values between the superior and temporal incision sub-groups [30].

Concerning different phacoemulsification techniques, surgeons should be aware that femtosecond laser-assisted cataract surgery (FLACS) can cause a higher incidence of postoperative dry eye symptoms than conventional phacoemulsification. Several authors have reported multiple intraoperative risk factors, including slower total operation time, exposure to microscopic light and laser energy power associated with this technique [31-33]. Those factors may alter limbal stem cells and conjunctival goblet cells (due to a suction ring) leading to decreased tear secretion and tear film stability. In addition, negative pressure caused by the suction ring used during femtosecond laser use can promote hyperaemia and inflammatory oedema, affecting the normal secretion of mucin and releasing many lysosomal enzymes and leukocytes [31-33].

Irrespective of the techniques used, it should be noted that phacoemulsification may modify 
meibomian gland function $[8,9,34,35]$. However, the exact mechanism is still unclear. Lid dysfunction due to the use of a speculum that alters the congruity and dynamics between the lid and ocular surface, a decrease in blinking rate secondary to altered corneal sensation, topical medications and the surgical procedure itself might influence ocular surface inflammation and development of DED that lead to functional changes in meibomian glands. As a result, MGD may be aggravated following cataract surgery with severity based on the preoperative MGD grade $[8,9,34,35]$.

\section{PREOPERATIVE MANAGEMENT}

Recently, several authors have suggested a number of criteria for determining the severity of DED and ocular surface disease before cataract surgery [36, 37]. However, a complete preoperative ocular surface assessment increases the visit time in an outpatient setting, which is not always practical. In a recent paper, the P.I.C.A.S.S.O. (Italian Partners for the Correction of Ocular Surface Alterations) board presented a timesaving method for a better management of DED [18]. In this paper, the P.I.C.A.S.S.O. board suggested a clinical evaluation could be achieved by considering the three pathogenic factors of DED: (1) tear film instability, (2) epithelial malfunction and/or suffering and (3) various degrees of clinically evident inflammation. These factors can be exacerbated by two other pathogenic findings: lid margin changes and nerve damage. This scheme has been named the " $3+2$ method" [18]. Using this method, we have created a comprehensive algorithm for the prevention, treatment and management of DED associated with cataract surgery, based on these five pathogenetic mechanisms (Fig. 2).

The preoperative examination involves the patients' history and evaluation of DED symptoms. Even if it is difficult to screen risk factors in routine clinical practice, surgeons should remember that DED is a multifactorial disorder that can be worsened by medical comorbidities such as diabetes and by certain classes of systemic medications including antihistamines, antidepressants, anxiolytics and isotretinoin, and topical medications including IOP-lowering drugs [3]. Large epidemiological studies confirm that being female or of older age increases the risk of DED [3, 38]. Other risk factors include ethnicity, MGD, pollution, low humidity and computer use [3]. Particular attention should also be paid to patients with Sjögren syndrome, rosacea, connective tissue diseases or haematopoietic stem cell transplantation [3]. A more general assessment of DED can be performed including questionnaires to quantify symptoms such as the Ocular Surface Disease Index (OSDI), 5-Item Dry Eye Questionnaire (DEQ-5), Symptom Assessment iN Dry Eye (SANDE) and Standard Patient Evaluation of Eye Dryness (SPEED) with the latest version SPEED II proposed by ASCRS [36].

Independent from the DED status, the minimum preoperative work-up should also comprise an accurate ocular surface examination, including fluorescein staining, TBUT and a complete evaluation of the ocular adnexa, conjunctiva and cornea. An impaired ocular surface will lead to inaccuracy in preoperative measurements such as keratometry and topography, which will affect the precision of intraocular lens (IOL) calculations. Therefore, surgeons should consider any dry eye-related alteration in preoperative measurements as a significant risk for a negative postoperative outcome [16]. If the results of the initial screening are not normal, further diagnostic tests can be performed to identify the subtype and severity of DED: Schirmer test, corneal sensitivity, non-invasive break-up time (NIBUT), tear meniscometry, meibography and point-of-care tests (POCTs) such as matrix metalloproteinase 9 and tear osmolarity [39].

The severity of the disease determines the preoperative management (Fig. 3). In mild forms of DED, surgery can be performed but patients should be prophylactically treated and informed about the possibility of postoperative persistence or aggravation of the symptoms. In uncontrolled or severe DED, surgery should be postponed until there is evidence of a significant improvement of symptoms, referring these cases to specialist centres on ocular surface diseases if long-term therapy is required [36, 37]. 


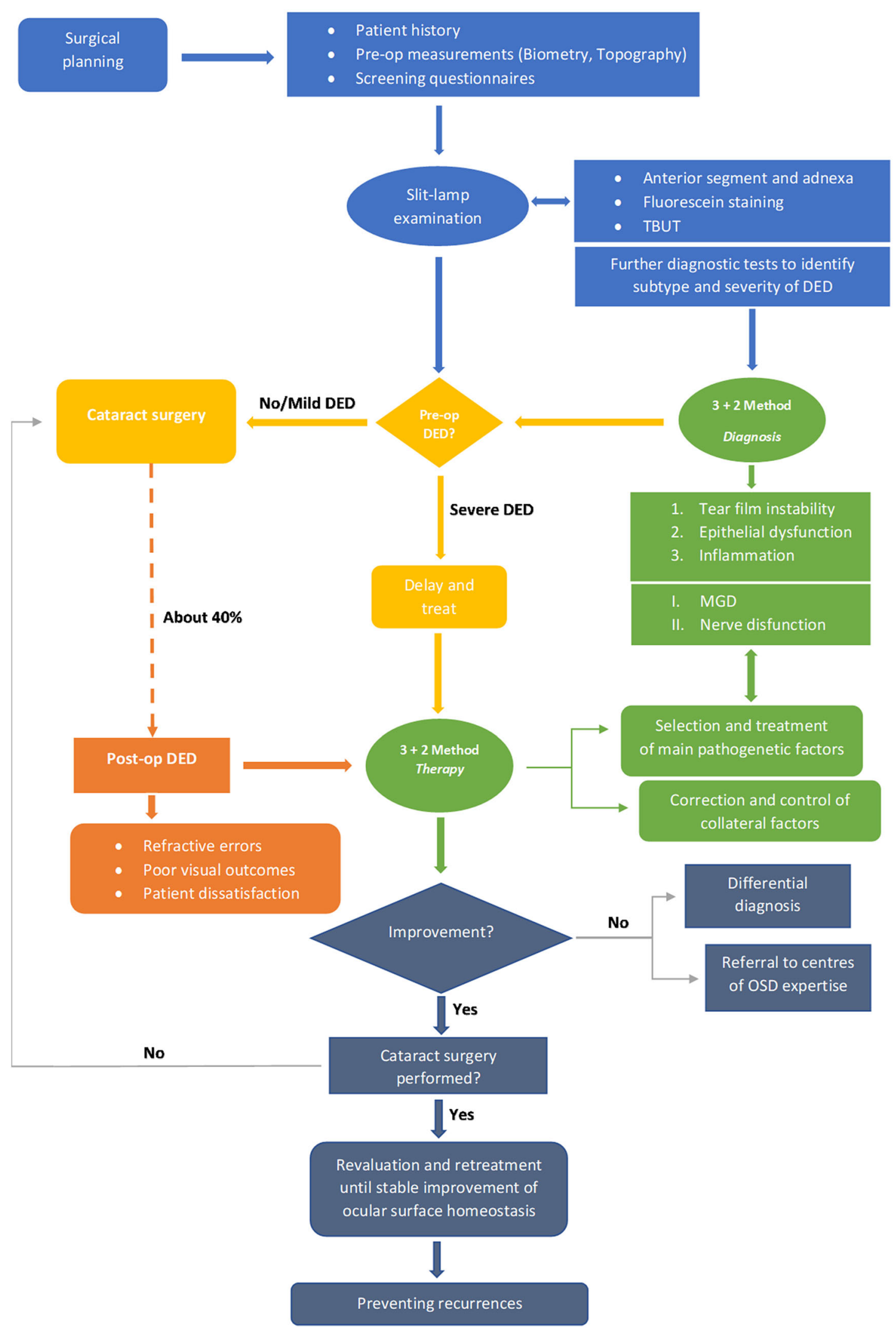


4Fig. 2 Algorithm to identify and manage patients affected by ocular surface disease that undergo cataract surgery through the evaluation of risk factors, correct diagnosis and an appropriate therapeutic strategy based on the $3+2$ method. DED dry eye disease, TBUT tear break up time, $M G D$ meibomian gland disfunction, $O S D$ ocular surface disease

However, ocular surface preparation is beneficial not only in patients with established DED, but also in those with minimal signs or symptoms. In a presurgical setting, rapid restoration of tear film homeostasis to minimise ocular surface complications should be targeted, which is generally achieved by a more aggressive, multifactorial approach. Artificial tears are the first choice of therapy for all forms of DED. For patients with MGD, lid hygiene, warm compresses and omega-3 fatty acid supplementation may also be suggested. When ocular surface inflammation is not sufficiently reduced in moderate or severe cases of DED, an adjunctive pulse therapy with topical steroids can be useful. Ciclosporin A and lifitegrast may also be proposed as steroid-sparing drugs [36, 40].

In the days preceding surgery, to further decrease the risk of postoperative complications, eye drops containing BAK should be avoided and replaced with preservative-free eye drops, if possible. In patients with severe DED, such as severe Sjögren syndrome, some studies

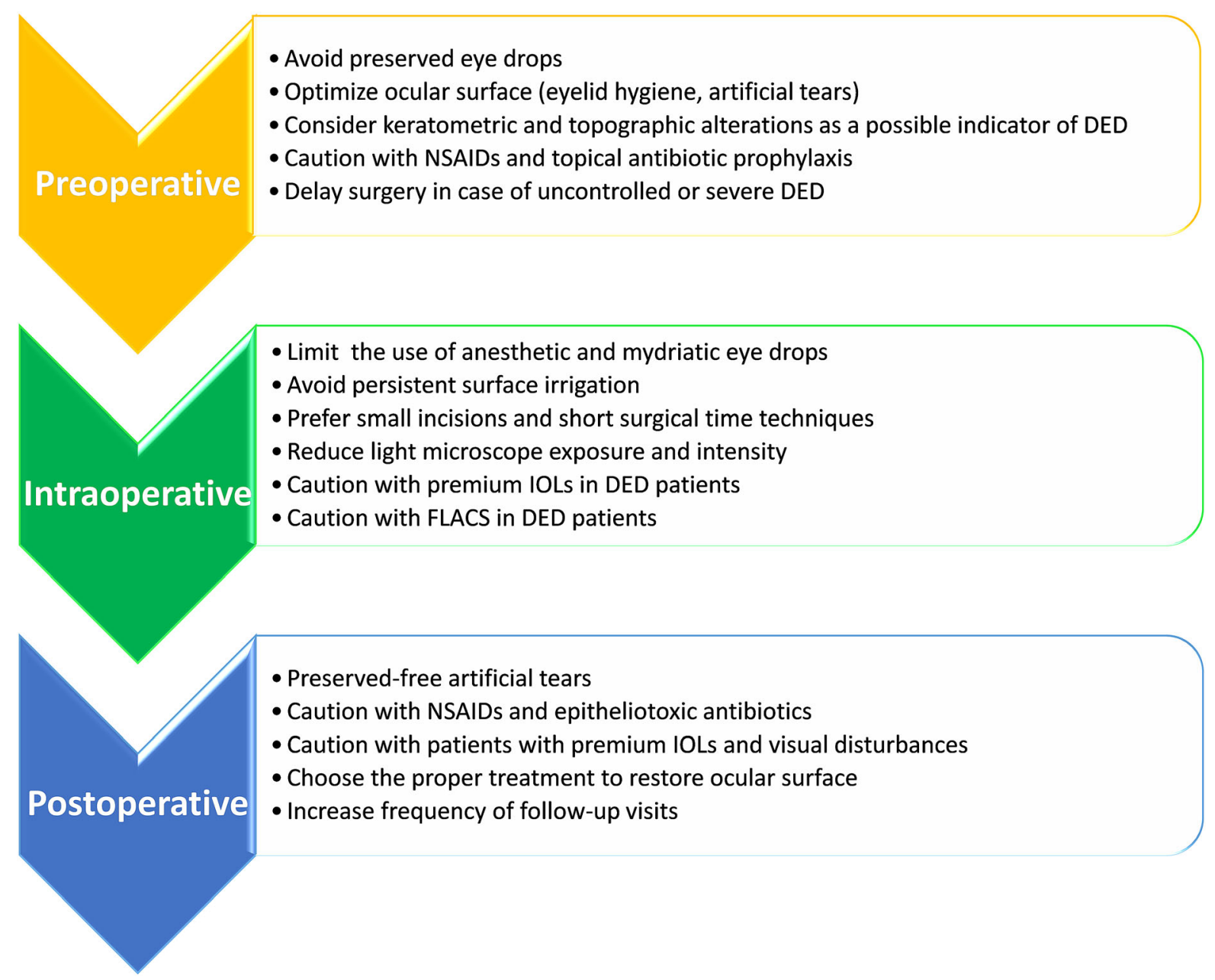

Fig. 3 Recommendations for preventing and reducing DED related to cataract surgery. NSAIDs nonsteroidal antiinflammatory drugs, FLACS femtosecond laser-assisted cataract surgery, $D E D$ dry eye disease, $I O L$ intraocular lens 
have suggested that the use of topical NSAIDs for prevention of postoperative cystoid macular oedema (CME) should be carefully evaluated because it may lead to neurotrophic keratitis and corneal melting $[41,42]$.

\section{INTRAOPERATIVE MANAGEMENT}

Different intraoperative factors can contribute to the vicious cycle of DED. Among them, the use of an aspirating speculum can induce or aggravate dry eye parameters. This can be the result of conjunctival damage, conjunctivochalasis and mechanical forces from the speculum that alter mucin secretion, increasing inflammation and impairing tear film stability [43]. Another contributing factor is povidone-iodine, which negatively alters the ocular surface. However, it is an essential aid in cataract surgery against postoperative endophthalmitis [37, 44, 45].

In patients with DED, anaesthetics and mydriatics should be administered by intracameral injection rather than eye drops to reduce ocular surface symptoms (Fig. 3). This was demonstrated in a phase III, randomised controlled study by Labetoulle et al. [46], where there were statistically significantly fewer patients who reported ocular surface symptoms in the group treated with an intracameral injection of a standardised ophthalmic combination of tropicamide $0.02 \%$, phenylephrine $0.31 \%$ and lidocaine $1 \%$ compared with the control group at 1 week and 1 month postoperatively: at 1 week postoperatively, only $0.7 \%$ of patients in the study group reported pain compared with the reference group $(3.9 \%)$ $(p=0.004) ;$ at 1 month, statistically significantly fewer patients reported irritation/burning/stinging in the study group (4.9\%) compared with the reference group (12.3\%) $(p=0.005)$ [46].

Moreover, a recently published study showed that the use of this intracameral solution during cataract surgery could reduce ocular surface inflammation evaluated by in vivo confocal microscopy, assuring a better postoperative tear film stability and visual quality [47]. A recent report [48] has also indicated that persistent intraoperative surface irrigation may contribute to immediate postoperative dry eye symptoms. Applying an ophthalmic viscosurgical device (OVD) on the corneal surface during surgery results in less ocular surface damage than balanced salt solution (BSS) irrigation on the surface, providing the advantage of a clear surgical view with no difference in surgical time $[48,49]$.

Other concerns relating to intraoperative management include light exposure, energy emitted during the procedure and incision size. Light exposure can be decreased by using appropriate filters and limiting exposure and intensity [30]. The correlation between phacoemulsification energy and DED is controversial. Cho and Kim [30] observed that phacoenergy did not exacerbate dry eye signs or symptoms. However, Sahu et al. [50] reported a negative influence, although this relationship was not statistically significant. Nevertheless, limiting the thermal energy delivered by phacoemulsification can contribute to a reduction in damage of the corneal structure, including the epithelium, stroma, keratocyte, endothelium and nerve plexus [30,50].

Finally, the risk of DED can be lowered by reducing the corneal incision size and the operation time. Therefore, small incision cataract surgery (SICS) and phacoemulsification might be comparable and represent an improvement in terms of ocular surface complications, while extracapsular cataract extraction (ECCE) theoretically results in a higher rate of postoperative DED [30, 51]. In addition, the presence of a corneal suture aggravates dry eye signs and symptoms [30]. As mentioned above, FLACS might have higher risk for exacerbating DED than standard phacoemulsification $[33,51]$.

\section{POSTOPERATIVE MANAGEMENT}

Several studies $[10,15,52]$ have demonstrated that homeostasis indicators such as corneal sensitivity, tear film stability and average density of goblet cells are reduced at 1 day postoperatively, with a peak at 7 days followed by a progressive recovery. Meanwhile, the dry eye 
symptoms increase and have been reported to persist for 1-3 months postoperatively. In severe cases, the ocular surface does not recover until 6 months postoperatively and may progress to chronic DED [10, 15, 52].

As already mentioned, the creation of incisions alters the corneal nerve supply, leading to a reduction in tear secretion and destabilisation of the ocular surface [49]. Furthermore, it can be hypothesised that, similar to what happens in refractive surgery, aberrant spontaneous nerve impulses may begin to fire, giving abnormal sensations, which the brain reads as ocular dryness [53]. Despite modern, less invasive techniques, a certain degree of inflammation in cataract surgery still occurs [28]. Several authors have reported that in the late postoperative phase the inflammatory mediators could induce the synthesis of several neurotrophic factors that stimulate the regeneration of corneal nerves. Therefore, it has been suggested that in many cases postoperative DED is a transient phenomenon, naturally recovering within a few months $[54,55]$.

As mentioned before, a discrepancy between signs and symptoms is common [11]. Postoperative DED symptoms may include eye fatigue, foreign body sensation, tearing and grittiness as well as frequent transient blurred vision [1]. However, severe forms of DED without symptoms are frequent, especially in high-risk patients with diabetes, Sjogren syndrome, graftversus-host disease (GVHD), Stevens-Johnson syndrome and severe ocular surface disease [3]. Consequently, patients should be screened for DED early after surgery to avoid severe postoperative complications that might include a higher risk of endophthalmitis [51].

Postoperative management can be guided by the severity of DED (Fig. 3). We propose a comprehensive approach to the diagnosis and treatment of postoperative DED based on the previously mentioned $3+2$ method (Fig. 2). According to this strategy the mainstay of the therapeutic management is to establish the key factors contributing to the vicious cycle of DED, inflammation, epithelial dysfunction, tear instability, nerve impairment and MGD, which represent the most important factors contributing to loss of ocular surface homeostasis.
The main pathogenetic factors should be identified and treated together with correction and control of the secondary collateral factors. During the follow-up it is essential to re-evaluate the patient until a stable improvement in ocular surface homeostasis has been obtained. If necessary, a therapeutic strategy to prevent recurrences should be carried out [56].

Preservative-free artificial tears and eyelid hygiene represent the mainstay of dry eye therapy and patients should be instructed to start treatment or continue the preoperative regimen $[18,40]$. Tear substitutes may play an important role in achieving the control of the inflammatory process in postoperative DED, improving tear fluid clearance and reducing the concentration of pro-inflammatory cytokines. Modern artificial tear substitutes also act on tear instability and hyperosmolarity, other pathogenic factors of DED [56]. Currently available formulations contain viscosity-enhancing agents including carbomer (polyacrylic acid), carboxymethyl cellulose (CMC), dextran, hyaluronic acid (HA), HP-guar, hydroxypropyl methylcellulose (HPMC), polyvinyl alcohol (PVA), polyvinylpyrrolidone (PVP) and polyethylene glycol, which enhance lubrication and prolong tear retention time on the ocular surface $[40,57]$.

The association between these agents and osmoprotectants such as L-carnitine, betaine and trehalose has additional beneficial effects on the treatment of DED compared to viscosityenhancing agents [58]. Trehalose is a widely used ocular pharmaceutical agent, which has been shown to preserve the integrity of corneal and conjunctival cells and their intracellular organelles through multiple mechanisms including control of inflammation, maintenance of homeostasis and protection against apoptosis [59-62]. A recent study has also described the newer phenomenon of autophagy induction, which provides an anti-inflammatory milieu to the ocular surface [62].

For a complete ocular surface treatment, MGD must be considered. MGD represents an important but frequently underestimated and undertreated factor that contributes to the vicious cycle of postoperative DED and it may be aggravated by cataract surgery $[8,9]$. There 
are many clinical treatments for MDG, including eyelid hygiene, warm compresses, meibomian gland expression, omega-3 supplementation and oral antibiotics [63]. However, these treatments only provide shortterm relief of the symptoms. One recent clinical trial has evaluated the use of pulsed light prior to cataract surgery in patients with MGD to prevent postoperative flare-ups and obtain more sustained visual acuity and ocular comfort [64].

As part of the overall surgical plan, the postoperative eyedrop therapy should be carefully modified in patients with DED. Post-surgical care implies the use of topical antibiotics, nonsteroidal anti-inflammatory drugs (NSAIDs) and steroids. The use of these eyedrops is associated with epithelial toxicity and worsening of goblet cell density. Therefore, in cases of postoperative DED, epitheliotoxic antibiotics should be avoided or used with caution and/or for a short duration [51, 65]. In case of severe inflammation of the ocular surface, more aggressive treatment, including pulse topical steroid therapy or even topical immunomodulatory drugs, can be considered $[37,66]$.

Patients should be monitored for recurrence of symptoms or development of complications after treatment. Increasing the frequency of follow-up visits is useful for determining whether DED therapy needs to be modified. Patients should be informed that ongoing treatment for DED must be maintained to optimise long-term visual outcomes. Particular attention should be reserved for unsatisfied postoperative patients with presbyopia correcting IOLs that might be more susceptible to visual disturbances because of poor tear film. In these subjects, DED should be identified and treated before considering other surgical options, such as Nd:YAG capsulotomy or an IOL exchange. Closer surveillance and long-term treatment are often required postoperatively $[17,36]$.

\section{CONCLUSION}

Cataract surgeons must be aware of the risk of postoperative DED. Any patient undergoing cataract surgery should be informed about the possibility of experiencing postoperative dry eye symptoms, which may vary in severity, even in uncomplicated procedures. The duration of these symptoms is usually limited to a couple of months; however, they can persist longer.

The presence of DED and its risk factors should be investigated before cataract surgery to avoid postoperative complications [52]. A proactive pathophysiological approach based on $3+2$ method is useful for a global evaluation and treatment of DED in both the pre- and postoperative phases. An appropriate and tailored therapy of the ocular surface will lead to an effective improvement in the quality of life of each surgical patient.

\section{ACKNOWLEDGEMENTS}

Funding. No funding or sponsorship was received for this study or publication of this article.

Authorship. All named authors meet the International Committee of Medical Journal Editors (ICMJE) criteria for authorship for this article, take responsibility for the integrity of the work as a whole, and have given their approval for this version to be published.

Disclosures. Rita Mencucci, Roberto Vignapiano, Pierangela Rubino, Eleonora Favuzza, Emilia Cantera, Pasquale Aragona and Maurizio Rolando have nothing to disclose.

Compliance with Ethics Guidelines. This article is based on previously conducted studies and does not contain any studies with human participants or animals performed by any of the authors.

Data Availability. Data sharing is not applicable to this article as no datasets were generated or analyzed during the current study.

Open Access. This article is licensed under a Creative Commons Attribution-NonCommercial 4.0 International License, which permits any non-commercial use, sharing, adaptation, distribution and reproduction in any medium 
or format, as long as you give appropriate credit to the original author(s) and the source, provide a link to the Creative Commons licence, and indicate if changes were made. The images or other third party material in this article are included in the article's Creative Commons licence, unless indicated otherwise in a credit line to the material. If material is not included in the article's Creative Commons licence and your intended use is not permitted by statutory regulation or exceeds the permitted use, you will need to obtain permission directly from the copyright holder. To view a copy of this licence, visit http://creativecommons.org/licenses/by$\mathrm{nc} / 4.0 /$.

\section{REFERENCES}

1. Sajnani R, Raia S, Gibbons A, et al. Epidemiology of persistent post-surgical pain manifesting as dry eyelike symptoms after cataract surgery. Cornea. 2018;37(12):1535-41.

2. Barabino S, Solignani F, Rolando M. Dry eye-like symptoms and signs after cataract surgery. Invest Ophthalmol Vis Sci. 2010;51(13):6254.

3. Stapleton F, Alves M, Bunya VY, et al. TFOS DEWS II Epidemiology Report. Ocul Surf. 2017;15(3): 334-65.

4. Xue-Min $\mathrm{L}$, Lizhong $\mathrm{H}$, Jinping $\mathrm{H}$, et al. Investigation of dry eye disease and analysis of the pathologic factors in patients after cataract surgery. Cornea. 2007;26:S16-20.

5. Liu Z, Luo L, Zhang Z. Tear film changes after phacoemulsification. Zhonghua Yan Ke Za Zhi. 2002;38(5):274-7.

6. Oh T, Jung Y, Chang D, et al. Changes in the tear film and ocular surface after cataract surgery. Jpn J Ophthalmol. 2012;56(2):113-8.

7. Bron AJ, de Paiva CS, Chauhan SK, et al. TFOS DEWS II pathophysiology report. Ocul Surf. 2017;15(3):438-510.

8. Cochener B, Cassan A, Omiel L. Prevalence of meibomian gland dysfunction at the time of cataract surgery. J Cataract Refract Surg. 2018;44(2): 144-8.
9. Han KE, Yoon SC, Ahn JM. Evaluation of dry eye and meibomian gland dysfunction after cataract surgery. Am J Ophthalmol. 2014;157(6):1144-50.

10. Barabino $S$, Labetoulle $M$, Rolando $M$, et al. Understanding symptoms and quality of life in patients with dry eye syndrome. Ocul Surf. 2016;14(3):365-76.

11. Hua R, Yao K, Hu Y. Discrepancy between subjectively reported symptoms and objectively measured clinical findings in dry eye: a population based analysis. BMJ Open. 2014;4(8):e005296.

12. Trattler WB, Majmudar PA, Donnenfeld ED. The prospective health assessment of cataract patients' ocular surface (PHACO) study: the effect of dry eye. Clin Ophthalmol. 2017;11:1423-30.

13. Gupta PK, Drinkwater OJ, VanDusen KW, et al. Prevalence of ocular surface dysfunction in patients presenting for cataract surgery evaluation. J Cataract Refract Surg. 2018;44(9):1090-6.

14. Park Y, Hwang HB, Kim HS. Observation of influence of cataract surgery on the ocular surface. PLoS ONE. 2016;11(10):e0152460.

15. Kasetsuwan N, Satitpitakul V, Changul T, et al. Incidence and pattern of dry eye after cataract surgery. PLoS ONE. 2013;8(11):e78657.

16. Epitropoulos AT, Matossian C, Berdy GJ, et al. Effect of tear osmolarity on repeatability of keratometry for cataract surgery planning. J Cataract Refract Surg. 2015;41(8):1672-7.

17. Gibbons A, Ali TK, Waren DP. Causes and correction of dissatisfaction after implantation of presbyopia-correcting intraocular lenses. Clin Ophthalmol. 2016;10:1965-70.

18. Rolando M, Cantera E, Mencucci R, et al. The correct diagnosis and therapeutic management of tear dysfunction: recommendations of the P.I.C.A.S.S.O. board. Int Ophthalmol. 2018;38(2):875-95.

19. Aragona P, Rania L, Puzzolo D. The role of inflammation, inflammatory cytokines, and ocular surface markers in dry eye disease. In: Lemp MA, Benitez del Castillo JM, editors. Ocular surface disorders. London: JP Medical Publishing; 2013. p. 55-68.

20. Rolando M, Refojo MF, Kenyon KR. Tear water evaporation and eye surface diseases. Ophthalmologica. 1985;190:147-9.

21. Mantelli F, Argueso P. Functions of ocular surface mucins in health and disease. Curr Opin Allergy Clin Immunol. 2008;8:477-83. 
22. Fraunfelder FT, Sciubba JJ, Mathers WD. The role of medications in causing dry eye. J Ophthalmol. 2012;2012:285851.

23. Patel M, Fraunfelder FW. Toxicity of topical ophthalmic anesthetics. Expert Opin Drug Metab Toxicol. 2013;9(8):983-8.

24. Sarkar J, Chaudhary S, Namavari A, et al. Corneal neurotoxicity due to topical benzalkonium chloride. Invest Ophthalmol Vis Sci. 2012;53(4): 1792-802.

25. Cha SH, Lee JS, Oum BS, et al. Corneal epithelial cellular dysfunction from benzalkonium chloride (BAC) in vitro. Clin Exp Ophthalmol. 2004;32(2): 180-4.

26. Ipek T, Hanga MP, Hartwig A, et al. Dry eye following cataract surgery: the effect of light exposure using an in-vitro model. Cont Lens Anterior Eye. 2018;41(1):128-31.

27. Hwang HB, Kim HS. Phototoxic effects of an operating microscope on the ocular surface and tear film. Cornea. 2014;33(1):82-90.

28. Gomes JAP, Azar DT, Baudouin C, et al. TFOS DEWS II iatrogenic report. Ocul Surf. 2017;15(3):511-38.

29. Khanal S, Tomlinson A, Esakowitz L, et al. Changes in corneal sensitivity and tear physiology after phacoemulsification. Ophthalmic Physiol Opt. 2008;28(2):127-34.

30. Cho YK, Kim MS. Dry eye after cataract surgery and associated intraoperative risk factors. Korean J Ophthalmol. 2009;23(2):65-73.

31. Shao D, Zhu X, Sun W, Cheng P, Chen W, Wang H. Effects of femtosecond laser-assisted cataract surgery on dry eye. Exp Ther Med. 2018;16(6):5073-8.

32. Yu Y, Hua $\mathrm{H}, \mathrm{Wu} \mathrm{M}$, et al. Evaluation of dry eye after femtosecond laser-assisted cataract surgery. J Cataract Refract Surg. 2015;41(12):2614-23.

33. Ju RH, Chen Y, Chen HS. Changes in ocular surface status and dry eye symptoms following femtosecond laser-assisted cataract surgery. Int J Ophthalmol. 2019;12(7):1122-6.

34. Baudouin C, Messmer EM, Aragona P, et al. Revisiting the vicious circle of dry eye disease: a focus on the pathophysiology of meibomian gland dysfunction. Br J Ophthalmol. 2016;100:300-6.

35. Crosby NJ, Shepherd D, Murray A. Mechanical testing of lid speculae and relationship to postoperative ptosis. Eye (Lond). 2013;27(9):1098-101.
36. Starr CE, Gupta PK, Farid M, et al. An algorithm for the preoperative diagnosis and treatment of ocular surface disorders. J Cataract Refract Surg. 2019;45(5):669-84.

37. Labetoulle M, Rousseau A, Baudouin C. Management of dry eye disease to optimize cataract surgery outcomes: Two tables for a daily clinical practice. J Fr Ophtalmol. 2019;42(8):907-12.

38. Moss SE, Klein R, Klein BEK. Prevalence of and risk factors for dry eye syndrome. Arch Ophthalmol. 2000;118(9):1264-8.

39. Wolffsohn JS, Arita R, Chalmers R, et al. TFOS DEWS II Diagnostic Methodology report. Ocul Surf. 2017;15(3):539-74.

40. Jones L, Downie LE, Korb D, et al. TFOS DEWS II Management and Therapy Report. Ocul Surf. 2017;15(3):575-628.

41. Kato K, Miyake K, Hirano K. Management of postoperative inflammation and dry eye after cataract surgery. Cornea. 2019;1:S25-33 ((38 Suppl.)).

42. Moss JM, Sanislo SR, Ta CN. A prospective randomized evaluation of topical gatifloxacin on conjunctival flora in patients undergoing intravitreal injections. Ophthalmology. 2009;116(8):1498-501.

43. Moon H, Yoon JH, Hyun SH. Short-term influence of aspirating speculum use on dry eye after cataract surgery: a prospective study. Cornea. 2014;33(4): 373-5.

44. Saedon H, Nosek J, Phillips J, et al. Ocular surface effects of repeated application of povidone iodine in patients receiving frequent intravitreal injections. Cutan Ocul Toxicol. 2017;36(4):343-6.

45. Golozar A, Chen Y, Lindsley K, et al. Identification and description of reliable evidence for 2016 American academy of ophthalmology preferred practice pattern guidelines for cataract in the adult eye. JAMA Ophthalmol. 2018;136(5):514-23.

46. Labetoulle M, Findl O, Malecaze F, et al. Evaluation of the efficacy and safety of a standardised intracameral combination of mydriatics and anaesthetics for cataract surgery. $\mathrm{Br} \mathrm{J}$ Ophthalmol. 2016;100(7):976-85.

47. Mencucci R, Favuzza E, Scali G, et al. Protecting the ocular surface at the time of cataract surgery: intracameral mydriatic and anaesthetic combination versus A standard topical protocol. Ophthalmol Ther. 2020. https://doi.org/10.1007/s40123020-00311-4.

48. Yoon DY, Kim JH, Jeon HS. Evaluation of the protective effect of an ophthalmic viscosurgical device 
on the ocular surface in dry eye patients during cataract surgery. Korean J Ophthalmol. 2019;33(5): 467-74.

49. Mencucci R, Cennamo M, Favuzza E, et al. Triphasic polymeric corneal coating gel versus a balanced salt solution irrigation during cataract surgery: a postoperative anterior segment optical coherence tomography analysis and confocal microscopy evaluation. J Cataract Refract Surg. 2019;45(8): 1148-55.

50. Sahu PK, Das GK, Malik A. Dry eye following phacoemulsification surgery and its relation to associated intraoperative risk factors. Middle East Afr J Ophthalmol. 2015;22(4):472-7.

51. Chuang J, Shih KC, Chan TC. Preoperative optimization of ocular surface disease before cataract surgery. J Cataract Refract Surg. 2017;43(12): 1596-607.

52. Xue W, Zhu M, Zhu B, et al. Long-term impact of dry eye symptoms on vision-related quality of life after phacoemulsification surgery. Int Ophthalmol. 2019;39:419-29.

53. Belmonte C. Eye dryness sensations after refractive surgery: impaired tear secretion or "phantom" cornea? J Refract Surg. 2007;23(6):598-602.

54. Dastjerdi MH, Dana R. Corneal nerve alterations in dry eye-associated ocular surface disease. Int Ophthalmol Clin. 2009;49(1):11-20.

55. Kohli P, Arya SK, Raj A, et al. Changes in ocular surface status after phacoemulsification in patients with senile cataract. Int Ophthalmol. 2019;39: 1345-53.

56. Aragona P, Giannaccare G, Mencucci R, et al. Modern approach to the treatment of dry eye, a complex multifactorial disease: a P.I.C.A.S.S.O. board review. Br J Ophthalmol. 2020;bjophthalmol2019-315747 (Online ahead of print).

57. Aragona $\mathrm{P}$, Simmons PA, Wang $\mathrm{H}$, Wang $\mathrm{T}$. Physicochemical properties of hyaluronic acid based lubricant eyedrops. Trans Vis Sci Technol. 2019;8:2.
58. Caretti L, La Gloria VA, Piermarocchi R, et al. Efficacy of carbomer sodium hyaluronate trehalose vs hyaluronic acid to improve tear film instability and ocular surface discomfort after cataract surgery. Clin Ophthalmol. 2019;13:1157-63.

59. Fariselli C, Giannaccare G, Fresina M, et al. Trehalose/hyaluronate eyedrops effects on ocular surface inflammatory markers and mucin expression in dry eye patients. Clin Ophthalmol. 2018;12: 1293-300.

60. Sarkar S, Davies JE, Huang Z, et al. Trehalose, a novel mTOR independent autophagy enhancer, accelerates the clearance of mutant huntingtin and alpha-synuclein. J Biol Chem. 2007;282:5641-52.

61. Matsuo T. Trehalose protects corneal epithelial cells from death by drying. Br J Ophthalmol. 2001;85(5): 610-2.

62. Panigrahi T, Shivakumar S, Shetty R, et al. Trehalose augments autophagy to mitigate stress induced inflammation in human corneal cells. Ocul Surf. 2019;17(4):699-713.

63. Sabeti S, Kheirkhah A, Yin J. Management of meibomian gland dysfunction: a review. Surv Ophthalmol. 2020;65(2):205-17.

64. Ge J, Liu N, Wang X. Evaluation of the efficacy of optimal pulsed technology treatment in patients with cataract and Meibomian gland dysfunction in the perioperative period. BMC Ophthalmol. 2020;20(1):111.

65. Fogagnolo P, Favuzza E, Marchina D, et al. New therapeutic strategy and innovative lubricating ophthalmic solution in minimizing dry eye disease associated with cataract surgery: a randomized prospective study. Adv Ther. 2020;37(4):1664-74.

66. Hamada S, Moore TC, Moore JE, et al. Assessment of the effect of cyclosporine-A $0.05 \%$ emulsion on the ocular surface and corneal sensation following cataract surgery. Cont Lens Anterior Eye. 2016;39(1): $15-9$. 